\title{
THE INVERSION OF CONVOLUTION TRANSFORMS \\ BY DIFFERENTIAL OPERATORS
}

CHARLES FOX

1. Introduction. Theories have recently been formulated, by Hirschman and Widder $[1 ; 2 ; 3]$, for the inversion of convolution transforms of the type

$$
f(x)=\int_{-\infty}^{\infty} G(x-t) \phi(t) d t
$$

Here

$$
G(t)=\int_{-i \infty}^{i \infty} \frac{e^{s t}}{E(s)} d s
$$

where

$$
E(s)=\prod_{n=1}^{\infty}\left(1-s^{2} a_{n}^{-2}\right)
$$

and

$$
\sum_{n=1}^{\infty} a_{n}^{-2}
$$

is convergent. These authors prove that (1) can be inverted by the differential operator

$$
E(D) f(x)=\lim _{m \rightarrow \infty} \prod_{n=1}^{m}\left(1-D^{2} a_{n}^{-2}\right) f(x)=\phi(x),
$$

where $D$ stands for differentiation with respect to $x$. They also consider other forms for $E(s)$, such as

$$
E(s)=e^{b \cdot} \prod_{n=1}^{\infty}\left(1-s / a_{n}\right) e^{s / a_{n}}
$$

with condition (4) satisfied, and similar convolution transforms.

The object of this paper is to generalize the form of $G(t)$ by replacing the exponential factor in the integral on the right of (2) by generalized Fourier kernels [4, chap. 8]. Owing to the complexity of the

Presented to the Society, September 3, 1953; received by the editors February 18, 1953 and, in revised form, April 8, 1953. 
formulae obtained I do not aim at complete generality and will confine myself largely to the case of the Bessel function kernel. I shall indicate briefly how this can be further generalized in $\$ 4$, after stating and proving the main theorem in $\$ 3$.

2. The Bessel function kernel. For our purposes it is more convenient to put (1) in a somewhat different form. We write

$$
x=\log u \text { and } t=-\log v
$$

in (1) and obtain, after some obvious changes,

$$
f_{1}(u)=\int_{0}^{\infty} g(u v) \phi_{1}(v) d v
$$

The kernel $g(u v)$ of (7) lends itself more readily to our discussion than does the kernel $G(x-t)$ of (1).

It is well known that if $J_{\nu}(x)$ and $Y_{\nu}(x)$ denote the standard solutions of the Bessel equation of order $\nu$, then $x^{1 / 2} J_{\nu}(u x)$ and $x^{1 / 2} Y_{\nu}(u x)$ both satisfy the equation

$$
\left\{D^{2}+\left(u^{2}-\frac{\nu^{2}-1 / 4}{x^{2}}\right)\right\} y=0,
$$

where $D$, here and in the rest of this paper, denotes differentiation with respect to $x$. Consider now the equation

$$
f(x)=\int_{0}^{\infty} \frac{(x u)^{1 / 2} J_{\nu}(x u)}{E(u)}\left\{\int_{0}^{\infty}(u v)^{1 / 2} J_{\nu}(u v) \phi(v) d v\right\} d u,
$$

where

$$
E(u)=\prod_{n=1}^{\infty}\left(1+u^{2} a_{n}^{-2}\right)
$$

and

$$
\sum_{n=1}^{\infty} a_{n}^{-2}
$$

is convergent. From (8) we have

$$
\left\{1-\frac{1}{a_{n}^{2}}\left(D^{2}-\frac{\nu^{2}-1 / 4}{x^{2}}\right)\right\}\left\{x^{1 / 2} J_{\nu}(x u)\right\}
$$

$$
=\left(1+\frac{u^{2}}{a_{n}^{2}}\right) x^{1 / 2} J_{\nu}(x u) .
$$


We now proceed formally and postpone the discussion of the analytical difficulties to $\$ 3$. By continuous application of (12) to (9) and by differentiating through the integral sign we have

$$
\begin{aligned}
\prod_{n=1}^{\infty}\left\{1-\frac{1}{a_{n}^{2}}\left(D^{2}\right.\right. & \left.\left.-\frac{\nu^{2}-1 / 4}{x^{2}}\right)\right\} f(x) \\
& =\int_{0}^{\infty}(x u)^{1 / 2} J_{\nu}(x u)\left\{\int_{0}^{\infty}(u v)^{1 / 2} J_{\nu}(u v) \phi(\nu) d v\right\} d u \\
& =\phi(x)
\end{aligned}
$$

by Hankel's theorem [5, p. 456].

Again, on changing the order of integration in (9), we have

$$
f(x)=\int_{0}^{\infty}\left\{\int_{0}^{\infty} \frac{u J_{\nu}(x u) J_{\nu}(u v)}{E(u)} d u\right\}(x v)^{1 / 2} \phi(v) d v .
$$

Thus, on writing

$$
K(x, v)=\int_{0}^{\infty} \frac{u J_{v}(x u) J_{v}(u v)}{E(u)} d u,
$$

we see that the equation

$$
f(x)=\int_{0}^{\infty} K(x, v)(x v)^{1 / 2} \phi(v) d v
$$

is inverted by the differential operator of (13).

When $\nu^{2}=1 / 4$ these results specialize to results equivalent to those of (1), (2), and (3).

The integral in (16) does not often reduce to a simple form. But in the special case when

$$
E(u)=1+u^{2} / a^{2}
$$

we have $[5, \$ 13.53]$, for $\nu>-1$,

$$
\int_{0}^{\infty} \frac{u}{\left(1+u^{2} / a^{2}\right)} J_{\nu}(x u) J_{\nu}(u v) d u= \begin{cases}a^{2} I_{\nu}(a x) K_{\nu}(a v), & x \leqq v, \\ a^{2} I_{\nu}(a v) K_{\nu}(a x), & x \geqq v .\end{cases}
$$

Here $I_{\nu}(x)$ and $K_{\nu}(x)$ are Bessel functions of purely imaginary argument, defined as follows $[5, \S 3.7]$

$$
I_{\nu}(x)=\sum_{n=0}^{\infty} \frac{(x / 2)^{2 n+\nu}}{n ! \Gamma(n+\nu+1)}, \quad K_{\nu}(x)=\frac{\pi}{2}\left(\frac{I_{-\nu}(x)-I_{\nu}(x)}{\sin \pi \nu}\right) .
$$

It follows that the inversion of 


$$
\begin{aligned}
f(x)= & \int_{0}^{x} a^{2} I_{\nu}(a v) K_{\nu}(a x)(x v)^{1 / 2} \phi(v) d v \\
& +\int_{x}^{\infty} a^{2} I_{\nu}(a x) K_{\nu}(a v)(x v)^{1 / 2} \phi(v) d v
\end{aligned}
$$

is given by

$$
\left\{1-\frac{1}{a^{2}}\left(D^{2}-\frac{\nu^{2}-1 / 4}{x^{2}}\right)\right\} f(x)=\phi(x) .
$$

This simplifies still further in the case when $\nu=1 / 2$ for we have

$$
I_{1 / 2}(x)=(2 / \pi x)^{1 / 2} \sinh x \text { and } K_{1 / 2}(x)=(\pi / 2 x)^{1 / 2} e^{-x} .
$$

(22) is then easily verified by direct differentiation.

3. Statement and proof of main theorem. In the following theorem the kernel $K(x, v)$ is defined by (16) and the function $E(u)$ by $(10)$.

If (i) $\nu \geqq-1 / 2$, (ii) $a_{n}$ is real for all positive integral values of $n$ and $\sum_{n=1}^{\infty} a_{n}^{-2}$ is convergent, (iii) $\phi(v) \in L(0, \infty)$, and (iv) $\phi(v)$ is of bounded variation in the neighborhood of $v=x$, then the integral transform

$$
f(x)=\int_{0}^{\infty} K(x, v)(x v)^{1 / 2} \phi(v) d v
$$

is inverted by the differential operator

$$
\begin{aligned}
\prod_{n=1}^{\infty}\left\{1-\frac{1}{a_{n}^{2}}\left(D^{2}-\frac{\nu^{2}-1 / 4}{x^{2}}\right)\right\} f(x) & \\
& =\frac{1}{2}\{\phi(x+0)+\phi(x-0)\} .
\end{aligned}
$$

The proof will allow for the possibility that $a_{m}=\infty$ for $m>m_{0}$, where $m$ and $m_{0}$ are positive integers and $m_{0} \geqq 1$. In this case $E(u)$ in (10) reduces to a finite product and we shall incidentally justify the example of $\S 2$ in which $E(u)$ is defined as in (18).

We commence the proof by noting that the asymptotic expansion of the Bessel function [5, chap. VII] is given by

$$
J_{\nu}(x) \sim\left(\frac{2}{\pi x}\right)^{1 / 2} \cos \left(x-\frac{1}{2} \nu \pi-\frac{1}{4} \pi\right)\left\{1+o\left(\frac{1}{x^{2}}\right)\right\} .
$$

Also, from the general theory of expansion of entire functions into infinite products, we know that there exist positive constants $M$ and $u_{0}$ such that for any positive integer $p$ we have $[3,82]$ 


$$
0<1 / E(u)<M / u^{p}, \quad 0<1 / E_{m}(u)<M / u^{p},
$$

where $u>u_{0}$ and

$$
E_{m}(u)=\prod_{n=m+1}^{\infty}\left(1+u^{2} a_{n}^{-2}\right)
$$

Since, from (ii), $E(u)$ is a convergent infinite product, we have.

$$
\lim _{n \rightarrow \infty} E_{m}(u)=1 \text {. }
$$

From (24) it is evident that $x^{1 / 2} J_{\nu}(x)$ behaves like $\sin x$ or $\cos x$ for large values of $x$. Hence, except for the factor $1 / E(u)$, the integrands in (9) behave in much the same way as the integrands in the well known sine and cosine Fourier transforms. If we differentiate (9) with respect to $x$ through the integral sign it follows from (iii), (24), and (25) that the resultant integral is uniformly convergent with respect to $x$. This is also true if we differentiate in this manner a finite number of times. Consequently, on applying the differential operator (12) $m$ times to (9), we conclude that

$$
\begin{aligned}
\prod_{n=1}^{m}\left\{1-\frac{1}{a_{n}^{2}}\left(D^{2}\right.\right. & \left.\left.-\frac{\nu^{2}-1 / 4}{x^{2}}\right)\right\} f(x) \\
& =\int_{0}^{\infty} \frac{(x u)^{1 / 2} J_{\nu}(x u)}{E_{m}(u)}\left\{\int_{0}^{\infty}(u v)^{1 / 2} J_{\nu}(u v) \phi(v) d v\right\} d u \\
& =\int_{0}^{\infty} P_{m}(x, u) d u
\end{aligned}
$$

say. We shall also write

$$
\begin{aligned}
\int_{0}^{\infty} P(x, u) d u & =\int_{0}^{\infty}(x u)^{1 / 2} J_{\nu}(x u)\left\{\int_{0}^{\infty}(u v)^{1 / 2} J_{\nu}(u v) \phi(v) d v\right\} d u \\
& =\phi(x)
\end{aligned}
$$

from (i), (iii), and (iv) by Hankel's theorem [5, \$14.4]. Hence

$$
\begin{aligned}
& \prod_{n=1}^{m}\left\{1-\frac{1}{a_{n}^{2}}\left(D^{2}-\frac{\nu^{2}-1 / 4}{x^{2}}\right)\right\} f(x)-\phi(x) \\
&=\int_{0}^{x}\left\{P_{m}(x, u)-P(x, u)\right\} d u+\int_{x}^{\infty} P_{m}(x, u) d u \\
&-\int_{x}^{\infty} P(x, u) d u .
\end{aligned}
$$


From (29) and the principle of convergence, given a positive $\epsilon$, however small, we can find an $X_{0}$ such that

$$
\left|\int_{Y}^{Z} P(x, u) d u\right|<\epsilon / 3,
$$

for all values of $Y$ and $Z$ satisfying the inequalities $Z>Y>X_{0}$. Evidently $E_{m}(u) \geqq 1$ and $E_{m}(u)$ decreases steadily as $u$ increases. We may therefore apply the second mean value theorem $[6, \S 4.14]$ and deduce that

$$
\left|\int_{Y}^{Z} P_{m}(x, u) d u\right|=\left|\frac{1}{E_{m}(Y)} \int_{Y}^{T} P(x, u) d u\right|<\epsilon / 3,
$$

where $Z>T>Y>X_{0}$. This choice of $X_{0}$ is the same as for (31) and so is independent of $m$. From (27) we see that for any prescribed $X$ an $m_{0}$ can be found such that the first integral of (30) is, in absolute value, less than $\epsilon / 3$ whenever $m>m_{0}$. To sum up, given any positive $\epsilon$ we can first choose $X$, independent of $m$, and then choose $m_{0}$ so that the absolute values of each of the integrals of (30) is less than $\epsilon / 3$ for $m>m_{0}$. We therefore have

$$
\lim _{m \rightarrow \infty} \prod_{n=1}^{m}\left\{1-\frac{1}{a_{n}^{2}}\left(D^{2}-\frac{\nu^{2}-1 / 4}{x^{2}}\right)\right\} f(x)=\phi(x),
$$

which establishes (14).

To complete the proof we must establish (17), i.e. justify the change in the order of integration in (9). Denote the integrand of (9) by $Q(x, u, v)$. From (iii) and (24) it follows that the integral with respect to $v$ in (9) is uniformly convergent with respect to $u$, in the interval $0 \leqq u \leqq U$, for any positive $U$. Hence

$$
\begin{aligned}
\int_{0}^{U} & \left\{\int_{0}^{\infty} Q(x, u, v) d v\right\} d u \\
& =\int_{0}^{\infty}\left\{\int_{0}^{U} Q(x, u, v) d u\right\} d v \\
& =\int_{0}^{\infty}\left\{\int_{0}^{\infty} Q(x, u, v) d u\right\} d v-\int_{0}^{\infty}\left\{\int_{0}^{\infty} Q(x, u, v) d u\right\} d v .
\end{aligned}
$$

From (24) and (25) it follows that a constant $K$ exists such that

$$
\left|\int_{0}^{\infty}\left\{\int_{U}^{\infty} Q(x, u, v) d u\right\} d v\right| \leqq \int_{0}^{\infty}|\phi(v)| d v \int_{U}^{\infty} \frac{K}{u^{p}} d u
$$


for sufficiently large $U$. If $E(u)$ is an infinite product, as in (10), $p$ can be any positive integer. If $a_{m}=\infty$ for $m>m_{0} \geqq 1$, i.e. $E(u)$ is a finite product, we still have $p \geqq 2$. From (iii) it then follows that

$$
\lim _{U \rightarrow \infty} \int_{0}^{\infty}\left\{\int_{U}^{\infty} Q(x, u, v) d u\right\} d v=0 .
$$

On applying this result to (35) we see that

$$
\lim _{v \rightarrow \infty} \int_{0}^{v}\left\{\int_{0}^{\infty} Q(x, u, v) d v\right\} d u=\int_{0}^{\infty}\left\{\int_{0}^{\infty} Q(x, u, v) d u\right\} d v .
$$

The change in the order of integration in (9) is thus justified and the inversion of $(A)$ by the differential operator $(B)$ is therefore established.

4. Generalization of previous results. The methods used in $\$ 3$ to invert

$$
f(x)=\int_{0}^{\infty} K(x, v) \phi(v) d v
$$

by a differential operator can be generalized if two essential requirements are satisfied. In the pair of reciprocal formulae

$$
g(x)=\int_{0}^{\infty} h(x u) f(u) d u ; \quad f(x)=\int_{0}^{\infty} k(x u) g(u) d u
$$

the functions $h(x)$ and $k(x)$ are known as generalized Fourier kernels and when $h(x)=k(x)$ they are said to be symmetrical. An account of these functions is given in Titchmarsh [4, chap. VIII]. We shall confine ourselves to the case of bounded symmetrical kernels whose Mellin transforms are products of gamma functions [4, §8.12]. For large values of $x$ the kernel $h(x)$ then behaves like $\cos x$ or $x^{1 / 2} J_{v}(x)$ and so, whenever necessary, the arguments of $\$ 3$ can be used to justify a change in the order of integration or in the order of integration and proceeding to a limit.

The first of these two requirements is that $K(x, v)$ in (39) should be of the form

$$
K(x, v)=\int_{0}^{\infty} \frac{h(x u) h(v u)}{E(u)} d u,
$$

where $h(x)$ is a symmetrical Fourier kernel and $E(u)$ is as defined in (10) with condition (11) satisfied.

The second of the two requirements is that $y=h(u x)$ should satisfy 
a differential equation of the type

$$
L(x, D) y=-u^{2} y .
$$

Here $L$ must be a function of $x$ and $D$, which denotes differentiation with respect to $x$, and must not contain $u$ or $y$. (42) is evidently a generalization of (8) and is a type of differential equation which frequently occurs in the theory of eigenfunction expansions. Many Fourier kernels beside the Bessel function satisfy differential equations such as (42).

Consider now the equation

$$
f(x)=\int_{0}^{\infty} \frac{h(x u)}{E(u)}\left\{\int_{0}^{\infty} h(u v) \phi(v) d v\right\} d u,
$$

where, in addition to the assumptions for $h(x)$ stated above, we assume that $\phi(v) \in L(0, \infty)$ and is of bounded variation near $v=x$. By using the arguments of $\$ 3$ we deduce that

$$
\begin{aligned}
\prod_{n=1}^{\infty}\left\{1-\frac{L(x, D)}{a_{n}^{2}}\right\} f(x) & =\int_{0}^{\infty} h(x u)\left\{\int_{0}^{\infty} h(u v) \phi(v) d v\right\} d u \\
& =\phi(x) .
\end{aligned}
$$

Equation (45) is deduced from (44) by means of the generalized Fourier theorem [4, p. 232]. On changing the order of integration in (43) and defining $K(x, v)$ as in (41) it follows that (39) is inverted by the differential operator of (45).

These results can be extended to the case of the unsymmetric Fourier kernel, i.e. when $h(x) \neq k(x)$ in (40), but such generalizations are necessarily intricate.

\section{REFERENCES}

1. I. I. Hirschman, Jr. and D. V. Widder, Generalized inversion for convolution transforms, Duke Math. J. vol. 17 (1950) pp. 391-402.

2. - Generalized inversion formulas for convolution transforms, Duke Math. J. vol. 15 (1948) pp. 659-696.

3. - The inversion of a generalized class of convolution transforms, Trans. Amer. Math. Soc. vol. 66 (1949) pp. 135-201.

4. E. C. Titchmarsh, The theory of the Fourier integral, Oxford University Press.

5. G. N. Watson, The theory of Bessel functions, Cambridge University Press.

6. E. T. Whittaker and G. N. Watson, Modern analysis, Cambridge University Press.

MCGILL UNIVERSITY 